\title{
The effect of the high-speed stream following the corotating interaction region on the geomagnetic activities
}

\author{
S. Watari \\ Communications Research Laboratory, 4-2-1 Nukuikita, Koganei, Tokyo 184, Japan
}

Received: 3 July 1996 / Received: 20 January 1997 / Accepted: 13 February 1997

\begin{abstract}
The high-speed stream following the corotating interaction regions (CIRs) was analyzed. As a result of the analysis, it is found that the geomagnetic field is continuously disturbed in the high-speed stream in question. The geomagnetic disturbances with long duration recurred several rotations between December 1993 and June 1994. These disturbances were associated with a large recurrent coronal hole expanding from the south pole of the Sun. High-speed solar wind from this coronal hole was observed by the IMP-8 satellite during this period. However, the observed intensities of the geomagnetic disturbances were different for each recurrent period. This is explained by the seasonal effect. The disturbed geomagnetic condition continued in the highspeed stream after the passage of the CIRs. The long duration of these disturbances can be explained by the continuous energy input into the Earth's magnetosphere from the high-speed regions following the CIRs. This kind of long-duration geomagnetic disturbance in association with coronal holes has been observed in the declining phase of other solar cycles. The relation between the coronal-hole area and the maximum solar-wind velocity is not good for the well-developed large coronal hole analyzed here.
\end{abstract}

\section{Introduction}

Some geomagnetic disturbances recur with about a 27day period during several solar rotations. Bartels (1934) gave the name M-regions to the solar sources of these recurrent geomagnetic disturbances. Now it is generally accepted that these 27-day recurrent geomagnetic disturbances are associated with corotating interaction regions (CIRs) formed between the high-speed solar wind emanating from coronal holes and the preceding slow solar wind. This is based on the results of several spacecraft observations (Neupert and Pizzo, 1974; Timothy et al., 1975; Nolte et al., 1976; Bohlin and
Sheeley, 1978; Broussard et al., 1978; Sheeley et al., 1976).

Nolte et al. (1976) noted that the velocity of the solar wind from coronal holes is roughly proportional to the area of the holes, and the holes located near the equator have more chance of affecting the Earth than polar ones. However, the recent analysis by Watari et al. (1995) showed that the relation between coronal-hole area and solar-wind velocity is more complicated than that derived by Nolte et al. (1976).

The recurrent disturbances caused by the CIRs are most remarkable during the declining phase of solar cycles when the coronal holes are largest and extend to the solar equator (Sheeley and Harvey, 1981; Watari, 1990).

According to Joselyn (1995), on average only $20 \%$ of the observed near-equatorial coronal holes (located within $30^{\circ}$ of the helioequator) are associated with geomagnetic storms $(A p$ index $\geq 30$; the $A p$ index is a planetary averaged daily geomagnetic index). Thomson $(1995 \mathrm{a}, \mathrm{b})$ also noted a poor correlation $(\leq 0.10)$ between individual coronal-hole parameters (e.g., area, polarity, and latitude) and $A p$ index.

To explain their results, we also have to consider the effect of the propagation between the Sun and Earth and the effect of coupling between solar wind and the magnetosphere as well as the coronal-hole parameters.

Considering the effect of propagation, it is difficult quantitatively to measure or estimate the following effect: the formation of southward interplanetary magnetic field (IMF) and high dynamic pressure in the CIRs during the propagation.

Several researchers (Pizzo, 1991, and references therein; Pizzo and Gosling, 1994) have studied the evolution of the CIRs by the computer simulation. The Ulysses observations showed the tilted latitudinal structure of the CIRs, and the tilt of the CIRs decreases with increasing distance from the Sun (Gosling et al., 1993; Riley et al., 1996). The signature of the CIRs is weak at around high heliographic latitude where the high-speed 
solar wind from the coronal hole is dominant (Phillips et al., 1994, 1995).

Considering the coupling effect, many researchers (Garrett et al., 1974; Burton et al., 1975; Iyemori et al., 1979; Akasofu, 1981; Baker, 1986; Fay et al., 1986; McPherron et al., 1986; Murayama, 1986; Feldstein, 1992; Price and Prichard, 1993) have studied on this subject. One problem is the nonlinear response of the magnetosphere (Klimas et al., 1996 and references therein); Vassiliadis et al. (1995) used the nonlinear filtering to describe it, and other researchers (Lundstedt, 1992; Lundstedt and Wintoft, 1994; Gleisner et al., 1996) applied the neural network to this problem. However, there is still room for improvement.

With respect to the seasonal dependence of the coupling effect, Sheeley and Harvey (1981) pointed out the presence of Russell-McPherron effect (Russell and McPherron, 1973) on the CIRs. Crooker and Cliver (1994, and references therein) and Crooker et al. (1996) noted the important role of the Russell-McPherron effect on both coronal streamers and CIRs. Joselyn (1995) noted that coronal holes of either polarity can be associated with storms throughout the year even though there is a tendency for negative polarity holes to be more geoeffective in the spring (February-April) and positive polarity holes to be more geoeffective during AugustOctober. This suggests that both propagation and coupling effects are important in estimating the relation between coronal-hole characteristics and the size of geomagnetic storms.

Recently, Lindsay et al. (1995) studied the statistics of solar-wind parameters, which were observed by the Pioneer Venus Orbiter (PVO) between 1979 and 1988, in coronal mass ejections (CMEs) and CIRs. They found that both CIRs and CMEs produce magnetic fields significantly larger than the normal IMF. The CIRs are often associated with the fluctuating north-south component $(B z)$ of the IMF and tend to produce larger dynamic pressures than the CMEs. According to Tsurutani et al. (1995a, and references therein), the increase of the IMF fluctuation is due to large-amplitude Alfvén waves within the body of the corotating streams. Ulysses also observed the large-amplitude IMF fluctuations in the high-speed stream (Tsurutani et al., 1995b).

There are many studies on the CIRs and their effect on the geomagnetic disturbances mentioned already. However, the effect of high-speed regions following the CIRs on the geomagnetic disturbances has never been fully examined before. There is a query as to how the high-speed regions after the passage of the CIRs affect the geomagnetic condition. Here, the above subject is discussed using the long-duration recurrent geomagnetic storms observed between December 1993 and June 1994.

The Dst index is used as an indicator of geomagnetic disturbances in this study. The index is a measure of variation in the geomagnetic field due to the equatorial ring current. It is calculated from the horizontal components at approximately four near-equatorial geomagnetic stations at hourly intervals. The relation between this index and solar-wind parameters is explained physically (Feldstein, 1992, and references therein). Burton et al. (1975) noted that there is an empirical relationship between the Dst index and solarwind parameters [e.g., velocity $(V)$, the north-south component of the IMF $(B z)$, and mass density $(\rho)$; the solar-wind electric field $(V B z)$ and the dynamic pressure $\left.\left(\rho V^{2} / 2\right)\right]$

\section{Observation}

Between December 1993 and June 1994 (Bartels rotation number 2910-2916), long-duration geomagnetic disturbances recurred several rotations associated with a large negative-polarity coronal hole extending from the south pole of the Sun [P. Lantos (Observatoire de ParisMeudon), private communication, 1994; Watari, 1995]. During this period, several satellite anomalies are reported in association with high-energy electrons. Baker et al. (1994, 1996) related these high-energy electrons with the high-speed stream from the welldeveloped coronal hole.

After the Skylab era the HeI 10830-nm line at Kitt Peak National Observatory was used to distinguish coronal holes. Since late 1991 there has been continuous solar observation by the Japan/US soft X-ray telescope (SXT) (Tsuneta et al., 1991) on board the Yohkoh satellite. HeI coronal holes are usually associated with the soft X-ray ones. However, the HeI coronal holes are smaller than the soft X-ray coronal holes and they show patchy structure (Watari et al., 1995). This perhaps reflects the expansion of the magnetic field in the coronal hole because HeI observes in lower solar atmosphere than soft X-ray. Here the SXT images are used to recognise coronal holes. Coronal holes are observed as a dark area in soft X-ray images because of their low density.

Figure 1 shows the time evolution of a coronal hole observed by the SXT between December 1993 and April 1994. The dark region extending from the south pole in the soft X-ray images is a coronal hole with negative polarity. This large well-developed coronal hole recurred during several rotations shown in Fig. 1. The white curves in Fig. 1 show the boundary of the coronal hole determined by the soft X-ray intensity.

Figure 2 shows the 1-h-averaged solar-wind velocity observed by the IMP-8 satellite during this period. The arrows in Fig. 2 note the periods of $\geq 500 \mathrm{~km} / \mathrm{s}$ solar wind in association with the southern coronal hole in Fig. 1. To examine further the effect of the recurrent streams, they are divided into CIRs (regions between two vertical solid lines shown in Fig. 2) and high-speed regions (HSRs: regions between two vertical dashed lines in Fig. 2) following the CIRs. Here, CIRs and HSRs are defined as below. CIRs are the regions with the density enhancement in the solar wind by compression between slow and fast solar wind. HSRs are the regions with $\geq 500 \mathrm{~km} / \mathrm{s}$ solar-wind speed after the CIR passage. There is a good correspondence between the southern coronal hole and the observed high-speed solar wind (see Figs. 1 and 2).

Figure 3 shows the Dst index during the same periods shown in Fig. 2. The arrows, the vertical solid 


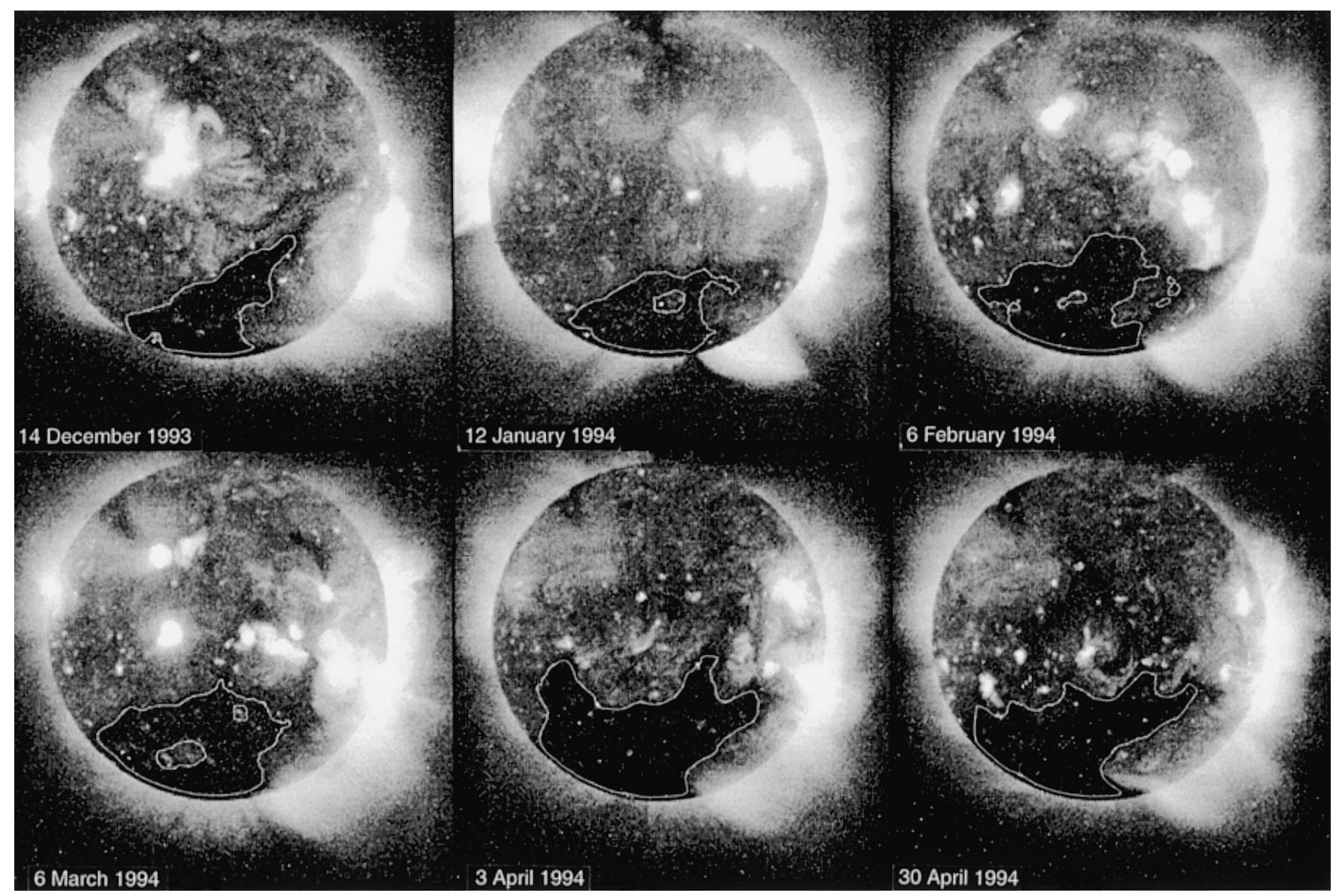

Fig. 1. Evolution of coronal holes observed by the soft X-ray telescope (SXT) on board the Japanese Yohkoh satellite. The white curves in the figure show the boundaries of the coronal holes determined by soft X-ray intensity

lines, and the vertical dashed lines in Fig. 3 correspond with those in Fig. 2. The disturbed geomagnetic condition continued after the passage of the CIRs, or in the HSRs. According to Figs. 2 and 3, the observed Dst variations were different even though the high-speed solar wind was observed associated with the coronal hole. This suggests that it is difficult to relate directly the coronal-hole parameters with the geomagnetic activities (Thomson, 1995a, b). The geomagnetic activities associated with the negative-polarity southern coronal hole are stronger and longer between February and April.

\section{Statistical analysis}

Table 1 summarizes the periods of $V \geq 500 \mathrm{~km} / \mathrm{s}$ of the solar wind associated with the south coronal hole with negative polarity, the maximum solar-wind velocity, and the ratios of the maximum velocity to background solarwind velocity (see the periods noted by the arrows in Fig. 2). Here, the low-speed solar wind in front of the CIRs is considered as the background solar wind. This table also shows the observed southern coronal hole areas between the helioequator and $30^{\circ}$ south from it

Table 1. Periods of high-speed streams associated with coronal holes, maximum solar-wind velocities, ratios of maximum to background solar-wind velocity, maximum Dst indices, and coronal-hole areas between the helioequator and $30^{\circ}$ south of it. ( $V$ back: background solarwind velocity; *: affected by data gaps.)

\begin{tabular}{|c|c|c|c|c|c|c|}
\hline Bartels rotation & $\begin{array}{l}\geq 500 \mathrm{~km} / \mathrm{s} \text { solar } \\
\text { start }(\mathrm{UT})\end{array}$ & $\begin{array}{l}\text { wind } \\
\text { end (UT) }\end{array}$ & $\begin{array}{l}\text { Max. velocity } \\
V \max (\mathrm{km} / \mathrm{s})\end{array}$ & $V \max / V$ back & $\begin{array}{l}\text { Max. Dst } \\
(\mathrm{nT})\end{array}$ & $\begin{array}{l}\text { Hole area between } 0 \\
\text { and } \mathrm{S} 30^{\circ}\left(10^{9} \mathrm{~km}^{2}\right)\end{array}$ \\
\hline 2190 & $93 / 12 / 16 / 0700$ & $93 / 12 / 22 *$ & 710 & 2.09 & -44 & 2.6 \\
\hline 2191 & $94 / 01 / 11 / 1300$ & $94 / 01 / 17^{*}$ & 775 & 2.58 & -42 & 4.0 \\
\hline 2192 & $94 / 02 / 06^{*}$ & $94 / 02 / 17^{*}$ & 805 & & -126 & 7.9 \\
\hline 2193 & $94 / 03 / 07 / 0900$ & $94 / 03 / 20 / 0500$ & 775 & 2.13 & -109 & 7.7 \\
\hline 2194 & $94 / 04 / 06^{*}$ & $94 / 04 / 13^{*}$ & 839 & $2.23^{*}$ & -111 & 10.0 \\
\hline 2195 & $94 / 05 / 01 / 1900$ & $94 / 05 / 10^{*}$ & 824 & $2.05^{*}$ & -79 & 4.0 \\
\hline 2196 & $94 / 05 / 28 / 1600$ & $94 / 06 / 09 / 0300$ & 786 & 2.07 & -68 & 4.2 \\
\hline
\end{tabular}



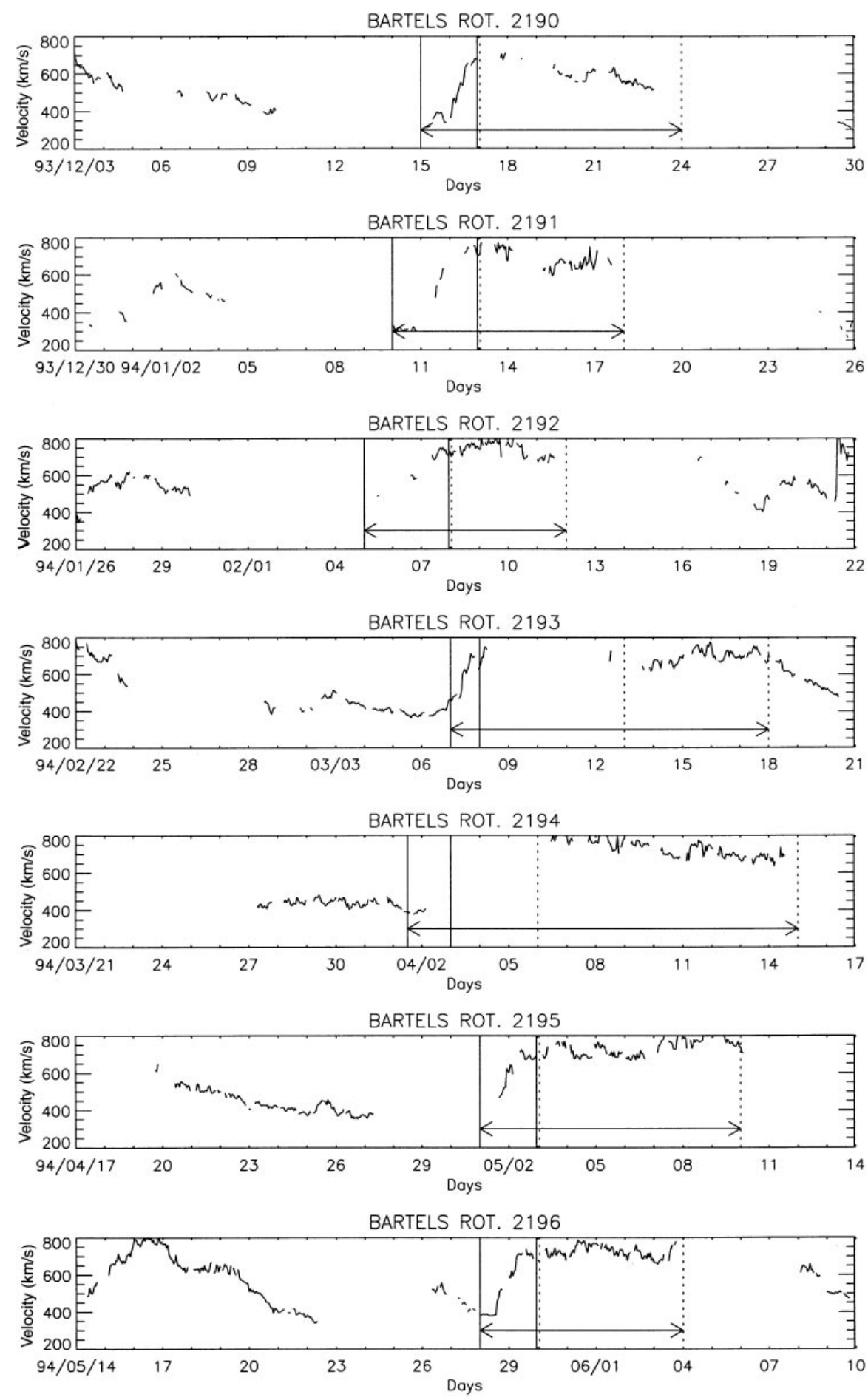

Fig. 2. Solar-wind velocity observed by IMP-8 between December 1993 and June 1994

(see Fig. 1) and the maximum Dst index during the recurrent geomagnetic disturbances in association with the coronal hole (see the periods noted by the arrows in Fig. 3). The coronal-hole areas were determined based on the soft X-ray images shown in Fig. 1.

High-speed streams with maximum velocity above $700 \mathrm{~km} / \mathrm{s}$ were observed associated with the large welldeveloped recurrent coronal hole for every rotation during this period. However, the relation between the coronal-hole area and the observed maximum velocity is not good (see Table 1). This result supports the recent result by Watari et al. (1995), and might suggest saturation, because all high-speed streams selected here exceed $700 \mathrm{~km} / \mathrm{s}$ at their maximum speed. Usually a high-speed stream associated with coronal holes does not exceed $850 \mathrm{~km} / \mathrm{s}$ at Earth.

The effect on geomagnetic disturbances (see maximum Dst index in Table 1) was different for each Bartels rotation. The interesting point is that the largest maximum Dst index in Table 1 is not associated with the highest maximum velocity and the largest coronal hole. The observed maximum velocities and Dst indices 

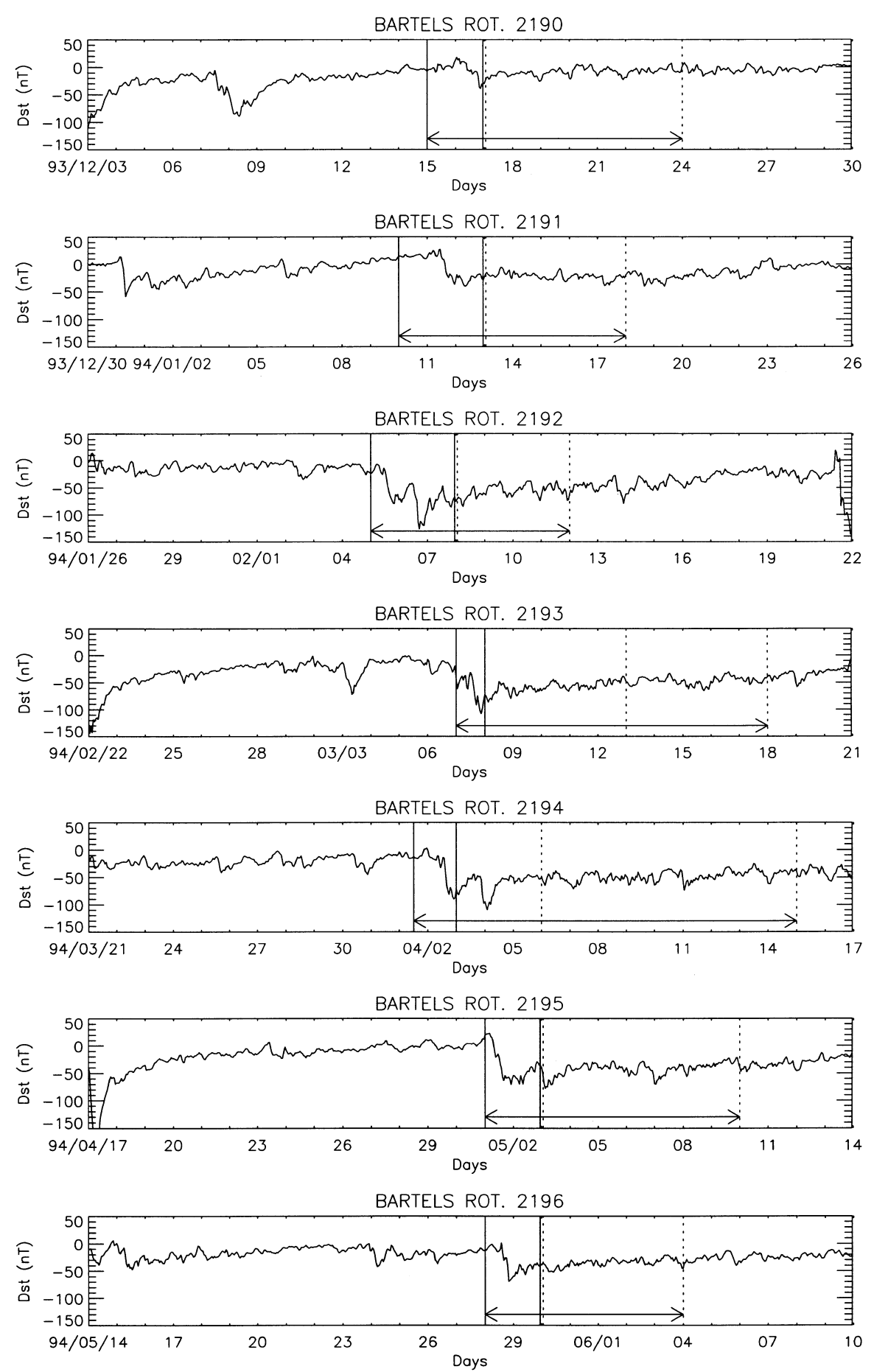

Fig. 3. Dst indices corresponding to the solar-wind observations shown in Fig. 2

are different even though the coronal holes are approximately same size $\left(\sim 4.0 \times 10^{9} \mathrm{~km}^{2}\right)$. The observed maximum Dst index in the Table 1 shows a larger value around March for the high-speed streams with approximately same maximum velocity $(\sim 775 \mathrm{~km} / \mathrm{s})$.

Table 2 shows the start, end, and duration of disturbed geomagnetic condition (Dst $\leq-15)$ in association with the high-speed stream from the south-pole coronal hole. The observed coronal-hole area and the maximum velocity differ for each Bartels rotation. However, it seems that the duration becomes longer in March and April.
Figure 4 shows the distribution of velocity $(V)$, number density $(n)$, temperature $(T)$, north-south component of IMF $(B z)$ in GSE and GSM, total flux of IMF $(|B|)$, solar-wind electric field $(V B z)$ in GSE and in GSM, dynamic pressure $\left(\rho V^{2} / 2\right)$ in the CIRs between December 1993 and June 1994 (Fig. 4a), in the HSRs (b), and in both the CIRs and the HSRs (c). The distribution is calculated for all 1-h-averaged data in the periods noted by the vertical solid lines (a), the vertical dashed lines (b), and the arrows (c) in Fig. 2.

Mass density $(\rho)$ is given by $m_{p} n$ and $m_{p}$ is proton mass. Two coordinate systems are used for $B z$. One is 
Table 2. Periods of Dst index of $\leq-15 \mathrm{nT}$ associated with coronal holes, maximum Dst indices, maximum solar-wind velocities, and coronal-hole areas between the helioequator and $30^{\circ}$ south of it

\begin{tabular}{|c|c|c|c|c|c|c|}
\hline Bartels rotation & $\begin{array}{l}\text { Dst } \leq-15(\mathrm{nT}) \\
\text { start (UT) }\end{array}$ & end (UT) & duration & $\begin{array}{l}\text { Max. Dst } \\
\text { (nT) }\end{array}$ & $\begin{array}{l}\text { Max. velocity } \\
(\mathrm{km} / \mathrm{s})\end{array}$ & $\begin{array}{l}\text { Hole area between } 0 \\
\text { and } S 30^{\circ}\left(10^{9} \mathrm{~km}^{2}\right)\end{array}$ \\
\hline 2190 & $93 / 12 / 16 / 1800$ & $93 / 12 / 17 / 0700$ & $14^{\mathrm{h}}$ & -44 & 710 & 2.6 \\
\hline 2191 & $94 / 01 / 11 / 1600$ & $94 / 01 / 13 / 0200$ & $1^{\mathrm{d}} 11^{\mathrm{h}}$ & -42 & 775 & 4.0 \\
\hline 2192 & $94 / 02 / 05 / 1200$ & $94 / 02 / 18 / 1200$ & $13^{\mathrm{d}} 01^{\mathrm{h}}$ & -126 & 805 & 7.9 \\
\hline 2193 & $94 / 03 / 06 / 1800$ & $94 / 03 / 20 / 2100$ & $14^{\mathrm{d}} 04^{\mathrm{h}}$ & -109 & 775 & 7.7 \\
\hline 2194 & $94 / 04 / 02 / 1400$ & $94 / 04 / 20 / 2000$ & $18^{\mathrm{d}} 08^{\mathrm{h}}$ & -111 & 839 & 10.0 \\
\hline 2195 & $94 / 05 / 01 / 1100$ & $94 / 05 / 12 / 2000$ & $11^{\mathrm{d}} 10^{\mathrm{h}}$ & -79 & 824 & 4.0 \\
\hline 2196 & $94 / 05 / 28 / 1500$ & $94 / 06 / 05 / 1100$ & $7^{\mathrm{d}} 21^{\mathrm{h}}$ & -68 & 786 & 4.2 \\
\hline
\end{tabular}

the geocentric solar-ecliptic (GSE) coordinate system. The other is the geocentric solar magnetosphere (GSM) coordinate system. $B z$ is perpendicular to the ecliptic plane in the GSE and parallel to the dipole axis of the Earth in the GSM. Bz in GSM minimizes the RussellMcPherron effect when we estimate the coupling effect between $B z$ and the magnetosphere.

Table 3 gives the average values and the standard deviations of solar-wind parameters. In CIRs and
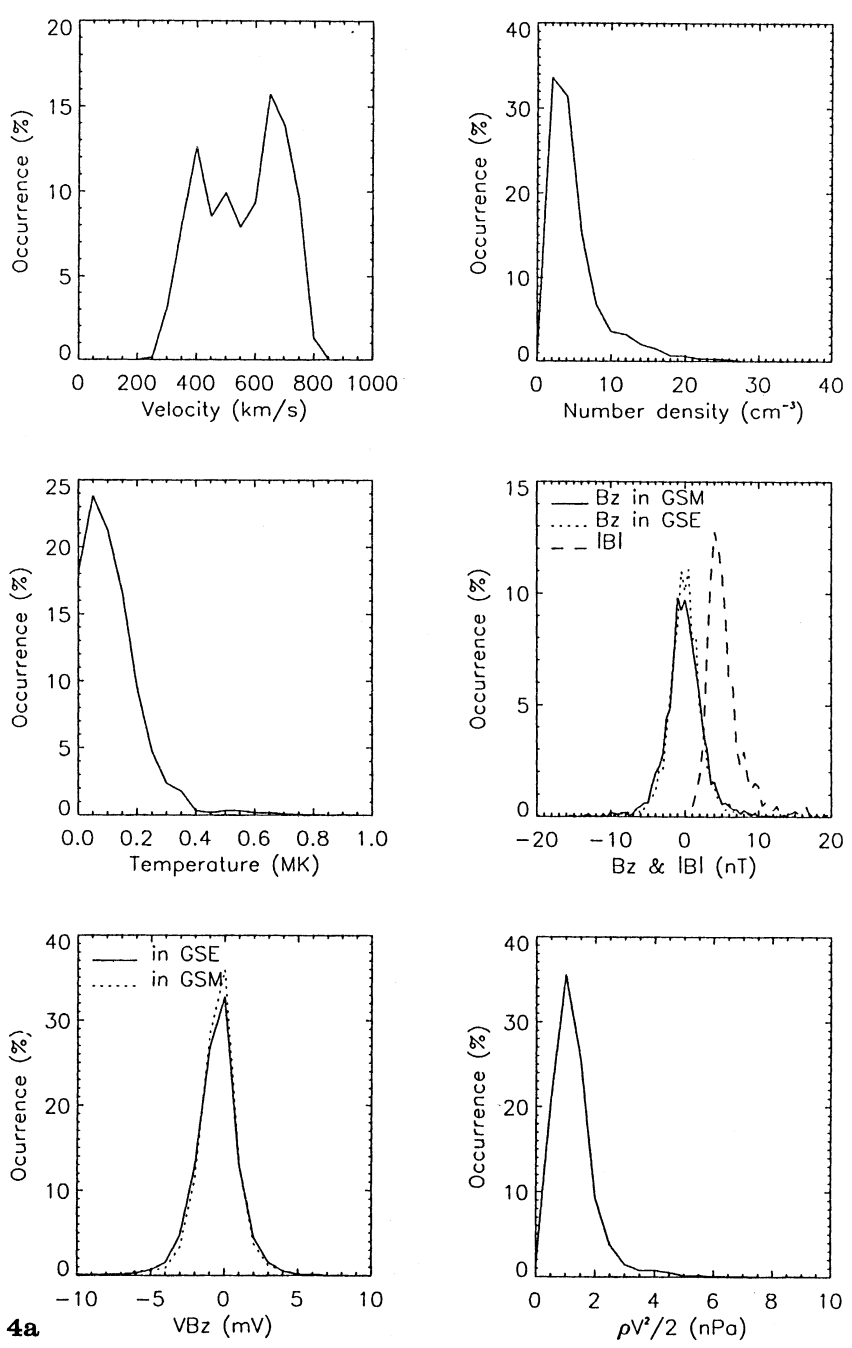
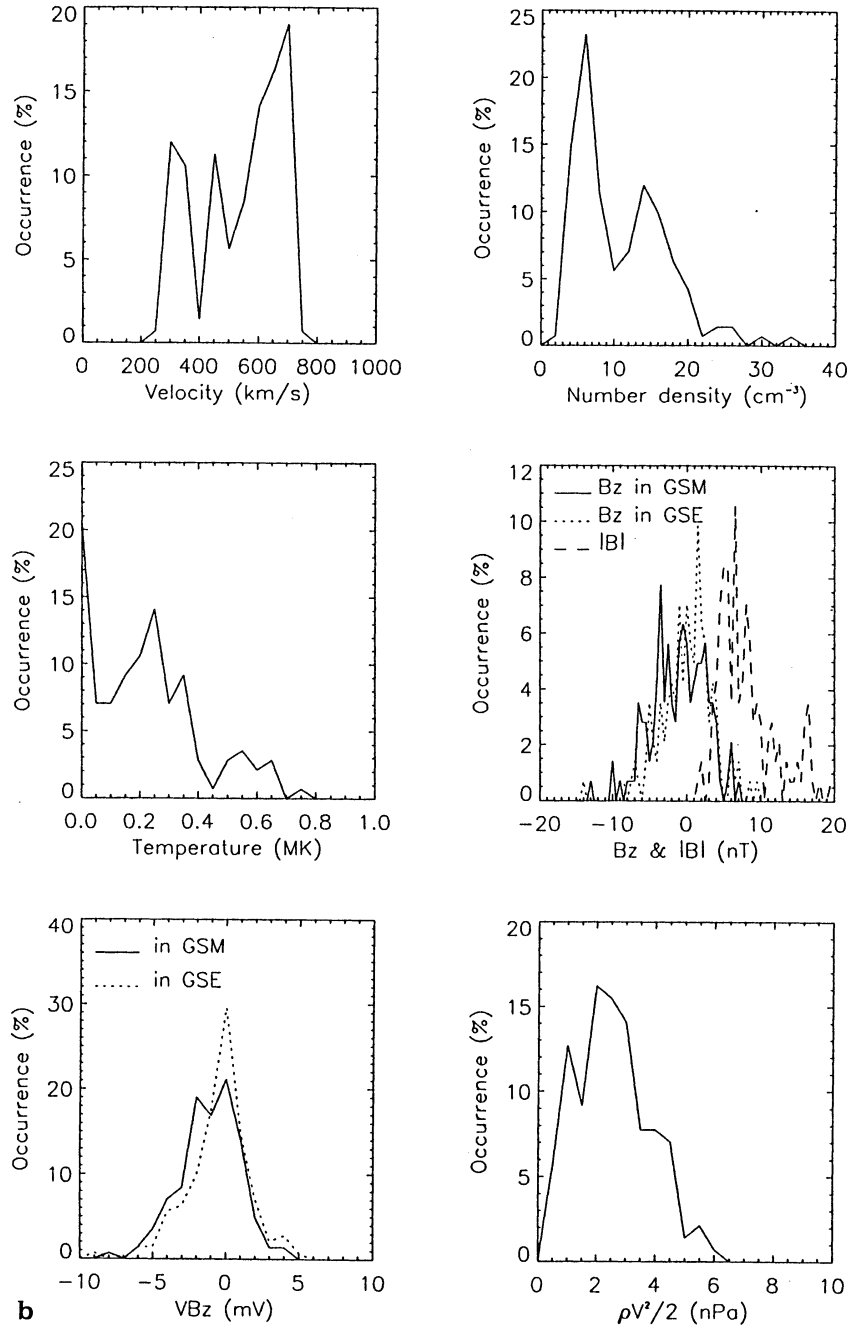

Fig. 4. a Distribution of solar-wind parameters in all the observed data. b Distribution of solar-wind parameters in the HDRs. $\mathbf{c}$ Distribution of solar-wind parameters in the HSRs 
Table 3. Average values and standard deviations of solar-wind parameters in HDRs, HSRs, and all observed solar wind

\begin{tabular}{lcrr}
\hline & in HDRs & \multicolumn{1}{l}{ in HSRs } & \multicolumn{1}{c}{$\begin{array}{l}\text { all observed } \\
\text { solar wind }\end{array}$} \\
\hline$V(\mathrm{~km} / \mathrm{s})$ & $559 \pm 145$ & $705 \pm 65$ & $581 \pm 136$ \\
$n\left(\mathrm{~cm}^{-3}\right)$ & $11.8 \pm 6.2$ & $4.0 \pm 1.1$ & $6.2 \pm 4.7$ \\
$T(\mathrm{MK})$ & $0.24 \pm 0.18$ & $0.18 \pm 0.10$ & $0.14 \pm 0.13$ \\
$|B|(\mathrm{nT})$ & $8.2 \pm 3.9$ & $4.9 \pm 1.2$ & $5.6 \pm 2.8$ \\
$B z$ in GSE (nT) & $-0.08 \pm 3.72$ & $-0.06 \pm 1.71$ & $0.17 \pm 2.52$ \\
$B z$ in GSM (nT) & $-0.91 \pm 3.65$ & $-0.55 \pm 1.80$ & $0.09 \pm 2.81$ \\
$V B z$ in GSE (mV) & $0.07 \pm 1.41$ & $0.02 \pm 2.12$ & $-0.06 \pm 1.22$ \\
$V B z$ in GSM (mV) & $-0.59 \pm 2.08$ & $-0.40 \pm 1.22$ & $-0.02 \pm 1.57$ \\
$\rho V^{2} / 2(\mathrm{nPa})$ & $2.80 \pm 1.26$ & $1.64 \pm 0.36$ & $1.58 \pm 1.67$ \\
\hline
\end{tabular}

creased in these regions (see Fig. 2). The larger electric field and dynamic pressure in the CIRs could control the magnitude of geomagnetic disturbances. The strong magnetic field with large fluctuation in the CIRs may reduce the seasonal dependence. The high dynamic pressure in the CIRs strongly compresses the magnetosphere and disturbs the geomagnetic field. This could also reduce the seasonal dependence.
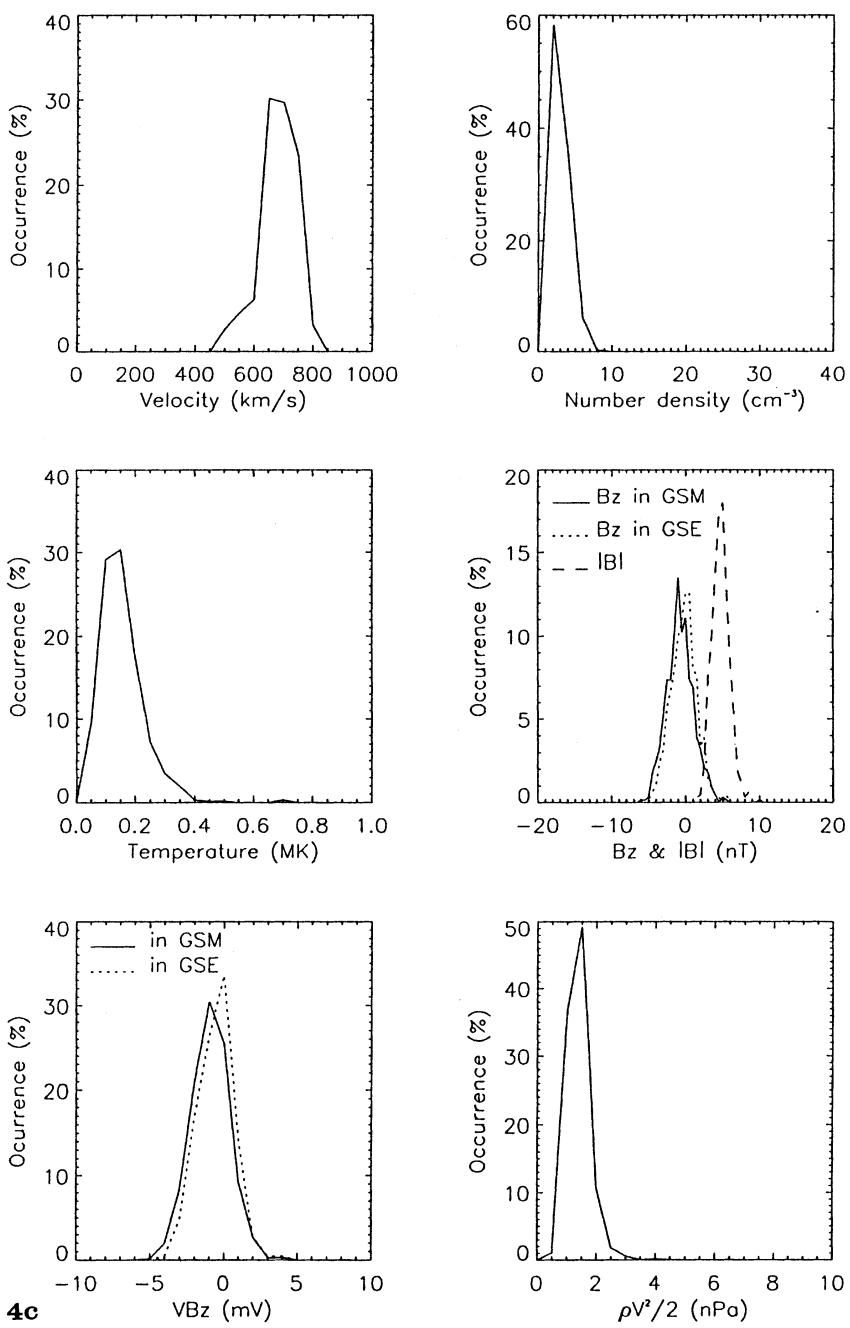

Fig. 4. $c$
Table 4. Correlation coefficients between Dst indices and solarwind parameters in the HDRs, HSRs, and all observed solar wind

\begin{tabular}{lrrr}
\hline & \multicolumn{2}{c}{ Correlation coefficients } & all observed solar wind \\
& in HDRs & in HSRs & \\
\hline$V$ & -0.70 & -0.55 & -0.58 \\
$n$ & 0.33 & 0.35 & 0.29 \\
$T$ & -0.59 & -0.18 & -0.29 \\
$|B|(\mathrm{nT})$ & -0.27 & -0.01 & -0.27 \\
$B z$ in GSE & 0.23 & 0.15 & 0.23 \\
$B z$ in GSM & 0.40 & 0.27 & 0.35 \\
$V B z$ in GSE & 0.20 & 0.14 & 0.24 \\
$V B z$ in GSM & 0.38 & 0.27 & 0.38 \\
$\rho V^{2} / 2$ & -0.45 & -0.10 & -0.13 \\
\hline
\end{tabular}

Table 4 shows the correlation coefficient between Dst indices and 1-h-averaged solar-wind parameters by the IMP-8 satellite in the CIRs, the HSRs, and both the CIRs and HSRs. All 1-h-averaged values in the periods noted by the arrows in Figs. 2 and 3 are used in calculating the correlation. The IMP-8 satellite is in near-circular 35-Earth radii orbit and the 1-h-averaged solar-wind parameters are used here. Hence, the difference of the location between the IMP-8 satellite and the magnetopause is neglected in the analysis. $B z$ and $V B z$ in GSM show better correlation than in GSE. This suggests the Russel-McPherron effect in both CIRs and HSRs. However, overall correlation between Dst indices and solar-wind parameters was very low except for velocity. The highest correlation $(-0.7)$ is with the velocity in CIRs.

\section{Concluding remarks}

The long-duration geomagnetic disturbances between December 1993 and June 1994 are analyzed. These disturbances are associated with the well-developed southern coronal hole. This recurrent hole was associated with high-speed solar wind of above $700 \mathrm{~km} / \mathrm{s}$ during this period. However, the size (magnitude and duration) of the geomagnetic disturbances was different for each rotation. It was found that the disturbed geomagnetic condition continues in the high-speed streams following the CIRs. They have the potential to keep a disturbed geomagnetic condition.

The coupling between the interplanetary magnetic field and geomagnetic field controls the magnitude and period of disturbed geomagnetic condition. The correlation between the Dst index and solar-wind parameters was low (less than 0.5 ), except for velocity. The average values of $n$ and $|B|$ and deviation of $|B|$ were larger in the CIRs because of compression (Lindsay et al., 1995). In CIRs, the Dst index showed good correlation with dynamic pressure. This is an explanation for the weak seasonal dependence of polarity of coronal holes in association with geomagnetic disturbances (Joselyn, 1995). In the HSRs, larger $V B z$ due to high velocity seems continuously to disturb the geomagnetic field. Watari (1990), Tsurutani et al. (1995a), and Crooker 
et al. (1996) noted the long duration geomagnetic disturbances in the declining phases of other solar cycles.

The relation between the coronal-holes area and maximum solar-wind speed is not good in this analysis. This might suggest saturation, because well-developed coronal holes are analyzed here.

Acknowledgments. This work was done as part of the Japan-France cooperation program. The author thanks Dr. P. Lantos (Observatoire de Paris-Meudon) for his suggestions and encouragement in this work. I thank NOAA/NGDC for Dst data, NASA/NSSDC for the IMP-8 data, and Yohkoh SXT-team for soft X-ray images.

Topical Editor R. Schwenn thanks S. Bravo and another referee for their help in evaluating this paper.

\section{References}

Akasofu, A. -I., Energy coupling between the solar wind and the magnetosphere, Space Sci. Rev., 28, 121, 1981.

Baker, D. N., Statistical analyses in the study of solar windmagnetosphere coupling, in Solar wind-magnetosphere coupling, Eds. Y. Kamide and J. A. Savin, Terra Scientific Publishing, Tokyo, pp. 17-38, 1986.

Baker, D. N., S. Kanekal, J. B. Blake, B. Klecker, and G. Rostoker, Satellite anomalies linked to electron increase in magnetosphere, EOS, 75, 401, 1994.

Baker, D. N., High-energy electrons in earth's magnetosphere: their effects and methods of prediction, in Proc. Solar-Terrestrial Prediction Workshop, Hitachi, Japan, in press, 1997.

Bartels, J., Twenty-seven day recurrences in terrestrial magnetic and solar activity, J. Geophys. Res., 37, 1, 1934.

Bohlin, J. D., and N. R. Sheeley Jr., Extreme ultraviolet observations of coronal holes, Solar Phys., 56, 125, 1978.

Broussard, R. M., N. R. Sheeley Jr., R. Tousey, and J. H. Underwood, A survey of coronal holes and their solar-wind associations throughout sunspot cycle 20, Solar Phys., 56, 161, 1978.

Burton, R. K., R. L. McPherron, and C. T. Russell, An empirical relationship between interplanetary condition and Dst, $J$. Geophys. Res., 80, 4204, 1975.

Crooker, N. U., and E. W. Cliver, Postmodern view of M-regions, J. Geophys. Res., 99, 13383, 1994.

Crooker, N. U., A. J. Lazarus, R. P. Lepping, K. W. Ogilvie, J. T. Steinberg, A. Szabo, and T. G. Onsager, A two-stream, foursector, recurrence pattern: implications from WIND for the 22year geomagnetic activity cycle, Geophys. Res. Lett., 23, 1275, 1996.

Fay, R. Y., C. R. Garrity, R. L. McPherron, and D. N. Baker, Prediction filters for the Dst index and the polar-cap potential, in Solar wind-magnetosphere coupling, Eds. Y. Kamide and J.A. Savin, Terra Scientific Publishing, Tokyo, pp. 111-118, 1986.

Feldstein, Y. I., Modelling of the magnetic field of magnetospheric ring current as a function of interplanetary medium parameter, Space Sci. Rev., 59, 83, 1992.

Garrett, H. B., A. J. Dessler, and T. W. Hill, Influence of solar wind variability on geomagnetic activity, J. Geophys. Res., 79, 4603, 1974.

Gleisner, H., H. Lundstedt, and P. Wintoft, Predicting geomagnetic storms from solar-wind data using time-delay neural networks, Ann. Geophysicae, 14, 679, 1996.

Gosling, J. T., S. J. Bame, D. J. McComas, J. L. Philips, V. J. Pizzo, and B. E. Goldstein, Latitudinal variation of solar wind corotating stream interaction regions: Ulysses, Geophys. Res. Lett., 20, 2789, 1993.

Iyemori, T., H. Maeda, and T. Kamei, Impulse response of geomagnetic indices to interplanetary magnetic fields, $J$. Geomagn. Geoelectr., 31, 1, 1979.
Joselyn, J. A., Geomagnetic activity forecasting: the state of the art, Rev. Geophys., 33, 383, 1995.

Klimas, A. J., D. Vassiliadis, D. N. Baker, and D. A. Roberts, The organized nonlinear dynamics of the magnetosphere, J. Geophys. Res., 101, 13089, 1996.

Lindsay, G. M., C. T. Rusell, and J. G. Luhmann, Coronal mass ejection and stream interaction region characteristics and their potential geomagnetic effectiveness, J. Geophys. Res., 100, $16999,1995$.

Lundstedt, H., Neural networks and predictions of solar-terrestrial effects, Planet. Space Sci., 40, 457, 1992.

Lundstedt, H., and P. Wintoft, Prediction of geomagnetic storms from solar-wind data with the use of a neural network, Ann. Geophysicae, 12, 19, 1994.

McPherron, R. L., D. N. Baker, and L. F. Bargatze, Linear filters as a method of real-time prediction of geomagnetic activity, in Solar wind-magnetosphere coupling, Eds. Y. Kamide and J. A. Savin, Terra Scientific Publishing, Tokyo, pp. 85-92, 1986.

Murayama, T., Coupling function between the solar wind and the Dst index, in Solar wind-magnetosphere coupling, Eds. Y. Kamide and J. A. Savin, Terra Scientific Publishing, Tokyo, pp. 119-126, 1986.

Neupert, W. M, and V. Pizzo, Solar coronal holes as sources of recurrent geomagnetic disturbances, J. Geophys. Res., 79, 253701, 1974.

Nolte, J. T., A. S. Krieger, A. F. Timothy, R. E. Gold, E. C. Roelof, G. Vaiana, A. J. Lazarus, J. D. Sullivan, and P. S. McIntosh, Coronal holes as sources of solar wind, Solar Phys., 46, 303, 1976.

Phillips, J. L., A. Baloph, S. J. Bame, B. E. Goldstein, J. T. Gosling, J. T. Hoeksema, D. J. McComas, M. Neugebauer, N. R. Sheeley Jr., and Y.-M. Wang, Ulysses at $50^{\circ}$ south: constant immersion in the high-speed solar wind, Geophys. Res. Lett., 21, 1105, 1994.

Phillips, J. L., S. J. Bame, W. C. Feldman, J. T. Gosling, C. M. Hammond, D. J. McComas, B. E. Goldstein, and M. Neugebauer, Ulysses solar-wind plasma observations during the declining phase of solar cycle 22, Adv. Space Res., 16, 85, 1995.

Pizzo, V. J., The evolution of corotating stream fronts near the ecliptic plane in the inner solar system, 2. Three-dimensional tilted-dipole fronts, J. Geophys. Res., 96, 5405, 1991.

Pizzo, V. J., and Gosling, J. T., 3D simulation of high-latitude interaction regions: comparison with Ulysses results, Geophys. Res. Lett., 21, 2063, 1994.

Price, C. P., and D. Prichard, The nonlinear response of the magnetosphere: 30 October 1978, Geophys. Res. Lett., 20, 771, 1993.

Riley, P., J. T. Gosling, L. A. Weiss, and V. J. Pizzo, The tilts of corotating interaction regions at midheliographic latitudes, J. Geophys. Res., 101, 24349, 1996.

Russell, C. T., and R. L. McPherron, Semiannual variation of geomagnetic activity, J. Geophys. Res., 78, 92, 1973.

Sheeley Jr., N. R. and J. W. Harvey, Coronal holes, solar wind streams, and recurrent geomagnetic disturbances during 1978 and 1979, Solar Phys., 70, 237, 1981.

Sheeley Jr., N. R., J. W. Harvey, and W. C. Feldman, Coronal holes, solar wind streams, and geomagnetic disturbances: 19731976, Solar Phys., 49, 271, 1976.

Thomson, A. W. P., Coronal hole observations and the prediction of the Ap geomagnetic index, Proc. IUGG XXI General Assembly, pp. B168, 1995a.

Thomson, A. W. P., A statistical relationship between coronal hole central meridian passage, J. Geomagn. Geoelectr., 47, 1263, 1995 b.

Timothy, A. F., A. S. Krieger, and G. S. Vaiana, The structure and evolution of coronal holes, Solar Phys., 42, 135, 1975.

Tsuneta, S., L. Acton, M. Bruner, J. Lemen, W. Brown, R. Caravalho, S. Freeland, B. Jurcevich, M. Morrison, Y. Ogawara, T. Hirayama, and J. Owens, Soft X-ray telescope for the SolarA mission, Solar Phys., 136, 37, 1991. 
Tsurutani, B. T., W. D. Gonzalez, A. L. C. Gonzalez, F. Tang, J. K. Arballo, and M. Okada, Interplanetary origin of geomagnetic activity in the declining phase of the solar cycle, J. Geophys. Res., 100, 21717, 1995a.

Tsurutani, B, T., C. M. Ho, J. K. Arballo, B. E. Goldstein, and A. Balogh, Large-amplitude IMF fluctuations, in corotating interaction regions: Ulysses at midlatitude, Geophys. Res. Lett., 22, 3397, 1995b.

Vassiliadis, D., A. J. Klimas, D. N. Baker, and D. A. Roberts, A description of the solar wind-magnetosphere coupling based on nonlinear filters, J. Geophys. Res., 100, 3495, 1995.
Watari, S., The latitudinal distribution of coronal holes and geomagnetic storms due to coronal holes, Proc. 1989 SolarTerrestrial Prediction Workshop, Edited by R. T. Thompson, D. G. Cole, P. J. Wilkinson, M. A. Shea, D. Smart, G. Heckman. Published by National Oceanic and Atmospheric Administration, Environmental Research Laboratories, Boulder, Colorado, Leura, Australia, 1, 627, 1990.

Watari, S., Soft X-ray coronal holes and interplanetary disturbances, Proc. IUGG Gen. Assem., pp. B168, 1995.

Watari, S., Y. Kozuka, M. Ohyama, and T. Watanabe, Soft X-ray coronal holes observed by the Yohkoh SXT, J. Geomagn. Geoelectr., 47, 1063, 1995. 